\title{
Oscillation Reduction Method for Fast Crane Operation
}

Thomas KUO ${ }^{1}$, Yu-Chou CHIANG ${ }^{2}$, Sheng-Yung $\mathrm{CHENG}^{3}$ and Shih-Chung Jessy KANG ${ }^{4 *}$

\author{
${ }^{1}$ Research Assistant, Department of Civil Engineering, National Taiwan University \\ ${ }^{2}$ Research Assistant, Department of Civil Engineering, National Taiwan University \\ ${ }^{3}$ Undergrad Student, Department of Civil Engineering, National Taiwan University \\ ${ }^{4}$ Professor, Department of Civil Engineering, National Taiwan University \\ *Corresponding author's e-mail: sckang@ntu.edu.tw
}

\begin{abstract}
Crane-related fatalities continue to occur in the construction industry according to recent surveys. This research provides a simple and safe oscillation reduction method for fast crane operation. We used two related acceleration and deceleration in piecewise fashion to control the crane. Our method is to reduce the sway angle and to reach high speed operation. We studied the method analytically, numerically and experimentally. We found analytically the operational time was approximately proportional to the square root of the operation distance; a fourfold increase in distance required only twofold increase in operational time. In comparing to a common method in $100 \mathrm{~m}$ operation distance, the numerical simulations suggest that the operation time is $25 \%$ faster when the maximum sway angle is the same, or the sway angle is $44 \%$ smaller when the operation time is the same.
\end{abstract}

\section{KEYWORDS}

crane control, oscillation reduction, open-loop system, KUKA experiment

\section{INTRODUCTION}

Cranes are one of the most heavily used and shared resources in construction sites. There are more than 125,000 cranes operating in the construction industry in the United States. One major challenge in crane operation is how to safely increase the operation speed of the crane. Fast crane operation may result in a large sway of the hanging object increasing the possibility of accidents. It is a challenge to maintain a high efficiency of crane operation as well as the safety of the site. One of the methods to ensure safe crane operation is oscillation control.

Automatic oscillation control systems have been widely investigated (Abdel-Rahman and Nayfesh, 2000 and 2001; Abdel-Rahman et al., 2003; Kang and Miranda, 2006 and 2008). Among these include sliding mode controls (Bartolini et al., 2002; Lee et al., 2006), back-stepping controls (d'Andréa-Novel and Coron, 2000) and input-shaping controls 
(Sorensen et al., 2007).

Previous methods have mainly focused on oscillation amplitude reduction at the end of the motion. Methods in general do not consider the sway angle reduction during the operation. Thus, the operation speed in the methods is often limited to avoid large sway angles during the operation. Our study seeks to develop a simple, fast method to have a controllable small sway angles in high-speed operation. We have reduced both the sway angle and the vibration frequency.

\section{PROPOSED THREE-STAGE CONTROL METHOD}

We accelerate the crane with a hanging object at a fixed sway angle for an arbitrary period of time to greatly enhance the operation speed. When we use additional acceleration to reduce the sway angle to zero, the high speed of operation is retained. With this idea, a greater constant speed of operation compared to previous methods can be achieved with a zero sway angle whilst maintaining operational efficiency and safety. The key of reduce the sway angle to zero is the timing and duration of acceleration to counter balance the sway. The $1 / 4$ and 1/8 factors come from maximizing the initial acceleration time to reach as high speed as possible. It is optimized in this sense.

A three-stage method for developing the crane control was proposed as shown in Figure 1. We will describe the method as follows:

(1) Piecewise acceleration stage: This stage included two accelerations, $a_{1}$ and $a_{2}$, that act in a piecewise fashion on the pivot of the pendulum. $a_{1}$ is an arbitrary choice by the user, while $a_{2}=g \cdot \tan \left(\sqrt{2} \cdot \tan ^{-1}\left(\frac{a_{1}}{g}\right)\right)$. The first acceleration speeds up the operation and the second introduced acceleration is then used to maintain the hanging objects at a controlled sway angle. Finally, we use the first acceleration again to sway the system back to a zero sway angle. More specifically, this stage can be broken down as a series of motions from point to point:

(i) Accelerating with $a_{1}$ for a quarter of the pendulum period, $T / 4$, where $T$ is the pendulum period.

(ii) Maintaining constant speed $a_{1} \cdot T / 4$ for the following eighth of the pendulum period $T / 8$.

(iii) Imposing acceleration $a_{2}$ over time interval $t_{2}$, where $t_{2}=\left(v_{\max }-a_{1} \cdot T / 2\right) / a_{2}$ and $v_{\max }$ is the desired maximum speed.

(iv) Maintaining constant speed $a_{1} \cdot T / 4+a_{2} \cdot t_{2}$ for the following one eighth of the pendulum period $T / 8$. 


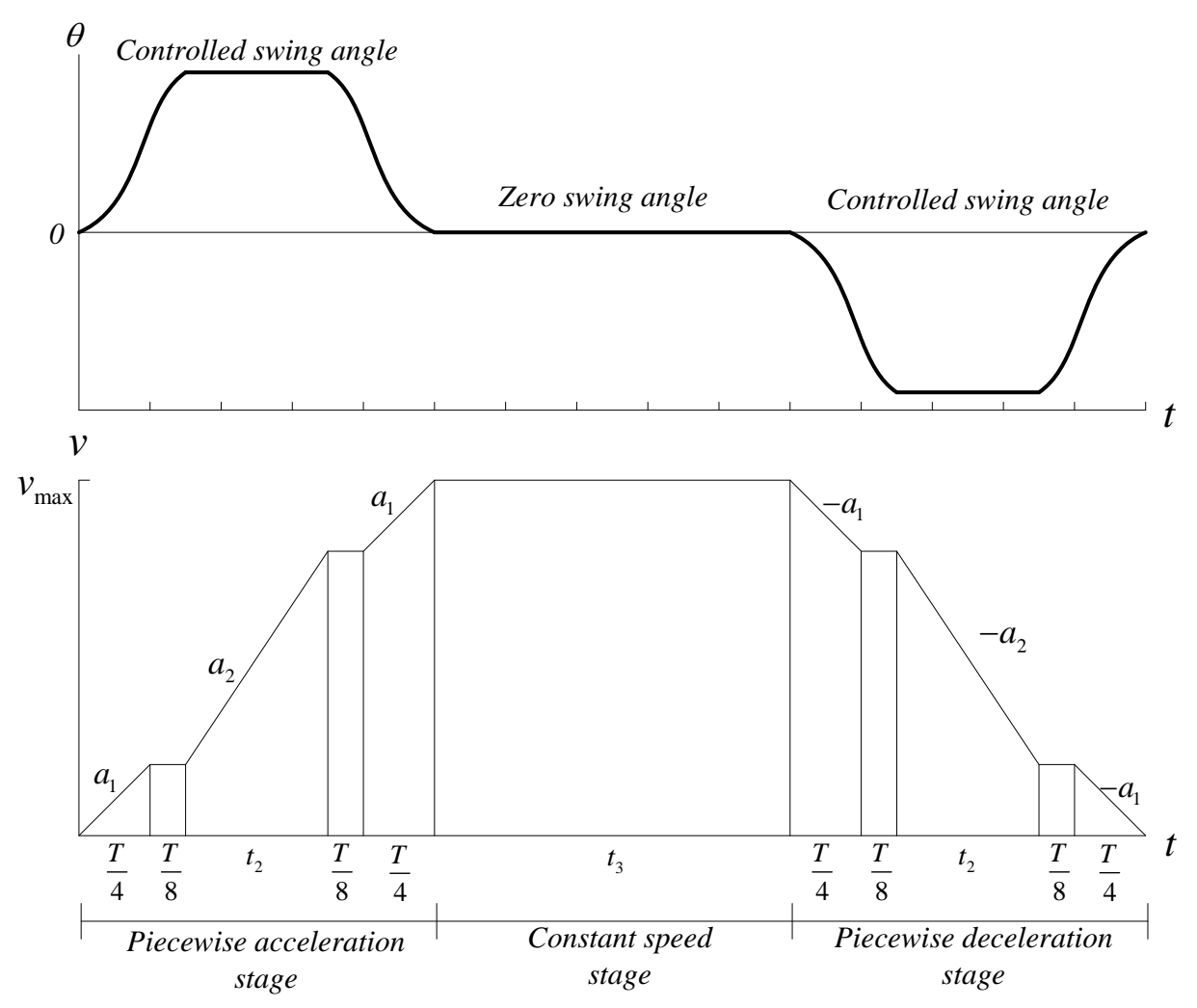

Figure 1. Schematic control diagram of the sway angle (upper) and velocity (lower) as a function of operational time.

(v) Accelerating with $a_{1}$ over a quarter of pendulum period $T / 4$ reaching the maximum operation speed $v_{\max }$.

(2) Constant speed stage: At this stage, no acceleration is applied and the pendulum moves at a constant speed $v_{\max }$ with zero sway angle.

(3) Piecewise deceleration stage: At this stage, the crane is decelerated to zero speed. The pattern of piecewise deceleration mirrors the first stage. Namely, $a_{1}$ and $a_{2}$ are replaced with $-a_{1}$ and $-a_{2}$ to completely stop the operation.

We conducted numerical experiments to validate the proposed three-stage control method which aims to produce smaller sway angles during high-speed operation. In addition to this, we further investigated the proposed method to develop a family of control plans based on the same principles.

Figure 2 is a schematic diagram for the control plan (a) and the special cases of $t_{2}=0$ (b), and $t_{3}=0$ (c) with the velocities as a function of time. The area below the velocity lines is the operation distance. For the sake of later discussion, we define the three plans in Fig. 2 as the A-plan, B-plan and C-plan, respectively. The A-plan in Fig. 2 (a) is the same as in Fig. 1. The A-plan is flexible with $t_{2}$ and $t_{3}$ parameters, and served as our generic control method. 
(a)

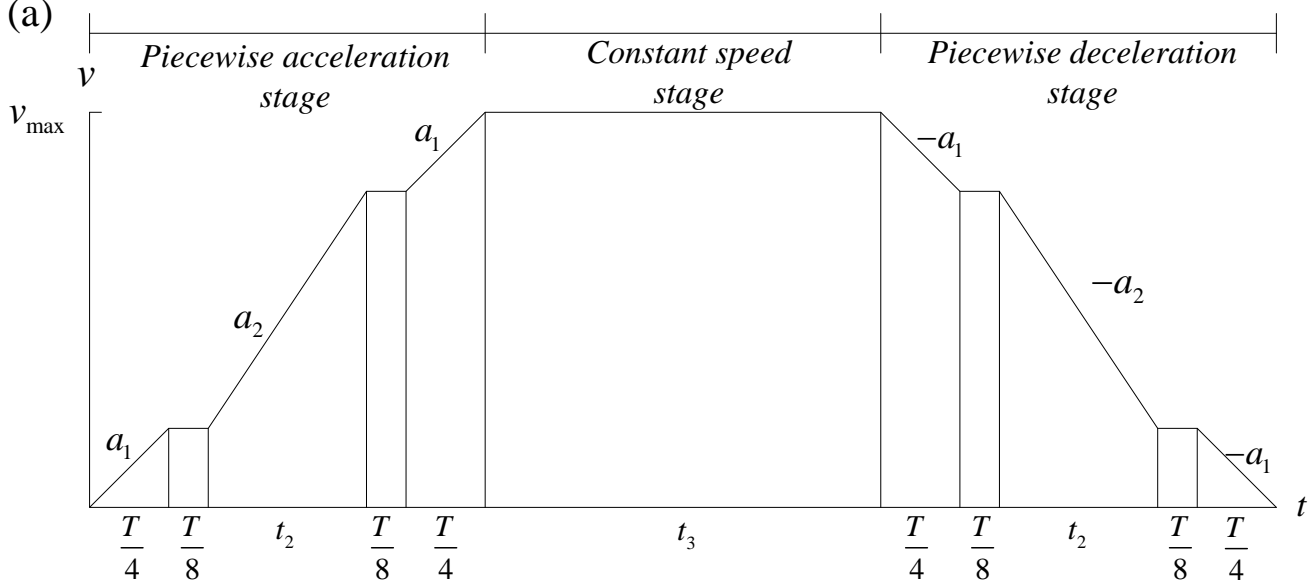

(b) $v$ Piecewise acceleration

Constant speed Piecewise deceleration

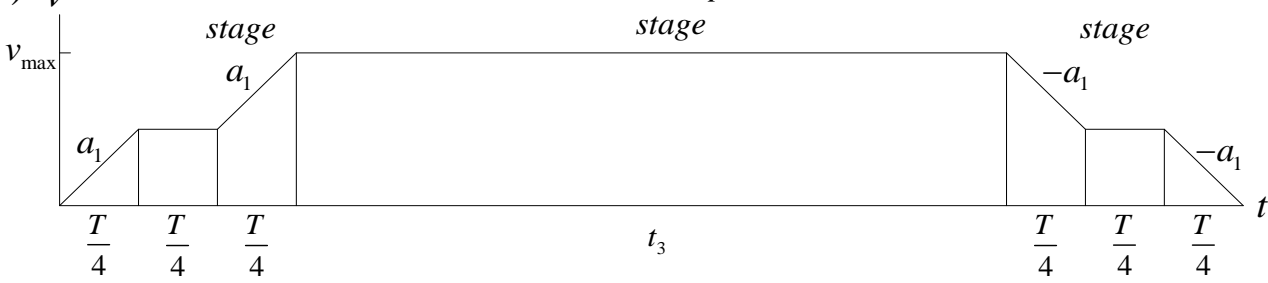

(c)

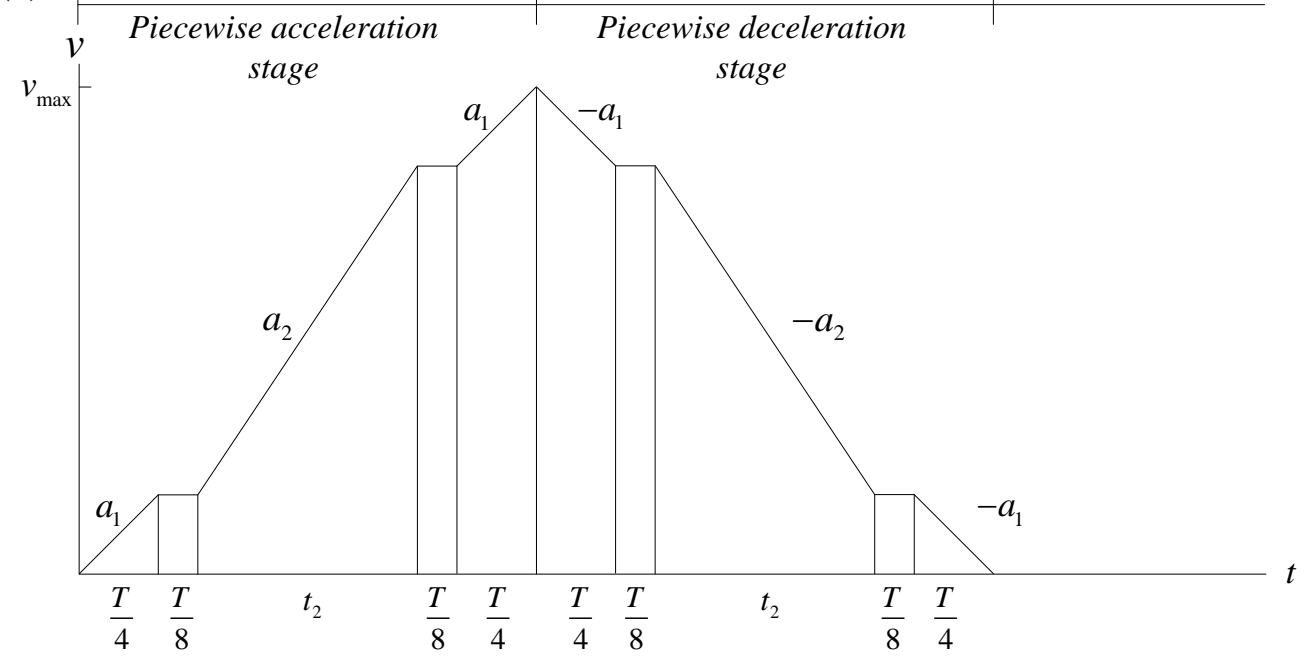

Figure 2. Similar to Fig. 1 the schematic diagram for velocity as a function of time of the A-plan (a), the B-plan with $t_{2}=0$ (b), and the C-plan with $t_{3}=0$ (c).

For a fixed operation distance, $d$, the B-plan is with constant speed and zero sway angle during most of the operation time. On the other hand, the C-plan $\left(t_{3}=0\right)$ is the fastest operating plan at the expense of not having a zero sway angle during the operation. The sway angle as a function of time for B-plan and C-plan is summarized in Figure 3 schematically.

With the same $a_{1}$ acceleration, the maximum sway angle was found to be the same for all 

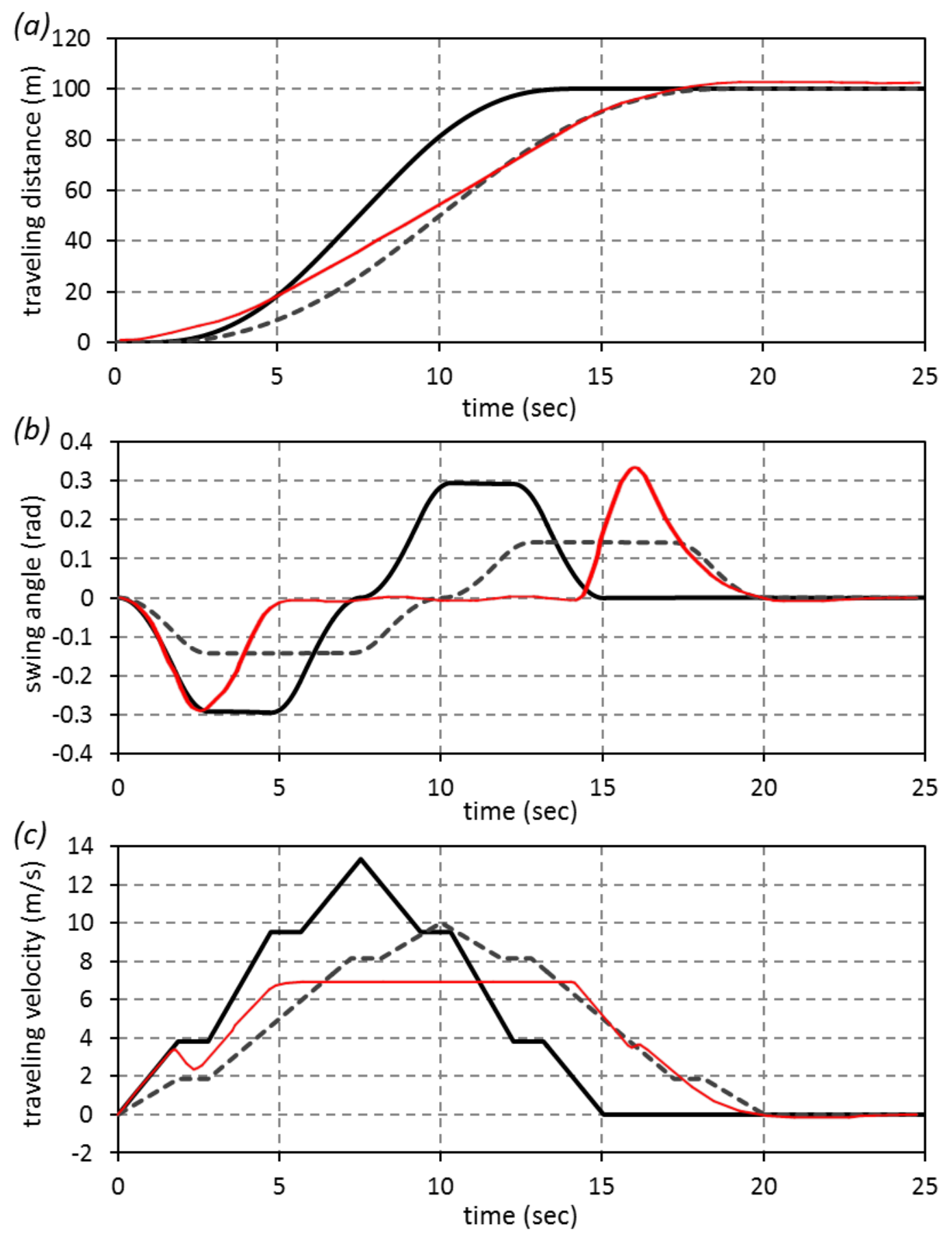

Figure 4. Numerical results of swift approach (black line), of small angle approach (dash line) and that of Lee and Cho (red lines) for a total operation distance of $100 \mathrm{~m}$. Traveling distance (a), sway angle (b), and input velocity (c) as a function of time.

To further investigate the operational time difference for the Normal-plan, B-plan, and the C-plan, we conducted a series of calculations with various operation distances. When the maximum sway angle is the same, the performance of the proposed method is better than the $\mathrm{N}$-plan for the operation distance greater than $0.74 \mathrm{~m}$ (Fig. 5). Figure 5 indicates that a fourfold increase in distance only required a $200 \%$ increase in operational time for C-plan. 


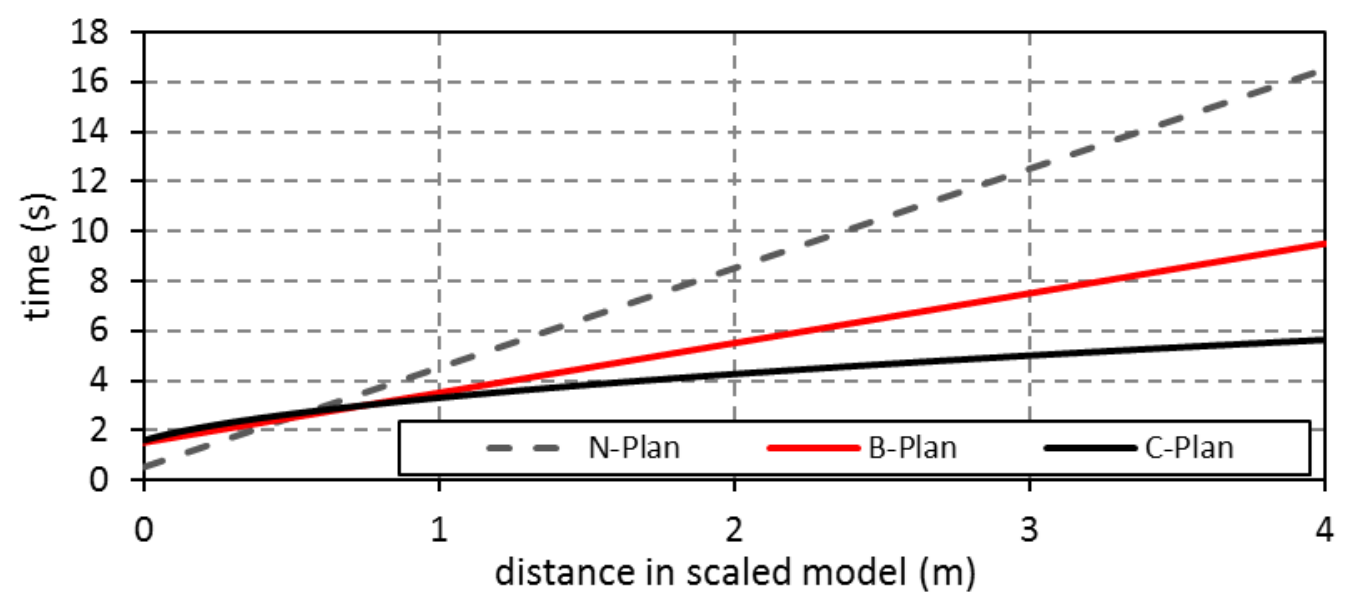

Figure 5. Operational distance as a function of time for the N-plan (grey dashed line), B-plan (red solid line) and C-Plan (black solid line).

This is equivalent to the operational time being roughly proportional to the square root of the distance increase. While the B-plan was slower than the C-plan, it was still considerably faster than the N-plan. Thus both plans were found to be suited for fast operation with long operation distances.

\section{CONCLUSION REMARKS}

We develop a three stage control method for fast crane operation based on double pendulum physics. The method use piece-wise acceleration and deceleration to reduce the sway angle and vibration frequency. We have demonstrated from the mathematical model, the operation time is proportional to the square root of the operation distance under a constant sway angle and no vibration. Our method is still in the early stage development as we have only demonstrated the physics and the advantages. We have conduct experiment using the method on KUKA robotic arm and scaled Tower crane in our lab. The sophisticate programed controller for the real operation has yet to be designed. In summary, we believe that our method is simple and efficient to control the sway of the crane cable. It is suitable to further develop into real crane operation.

\section{ACKNOWLEDGMENT}

The research was supported by the National Science Council of Taiwan through Grants NSC101-2221-E-002-185-MY3. This research was performed while the first author was a master student at National Taiwan University. We would like to thank Hung-Chi Kuo, Yu-Chou Chiang, and anonymous reviewers for their helpful comments. 


\section{REFERENCES}

Abdel-Rahman, E. M., Nayfeh, A. H. (2000). "Cargo-pendulation reduction in boom cranes via cable-length manipulation." in: Proceedings of the SDM 41th Annual Meeting, Atlanta, GA, April 03-06.

Abdel-Rahman, E. M., Nayfeh, A. H. (2001). "Feasibility of two-dimensional control for ship-mounted cranes." in: Proceedings of the ASME DETC, Pittsburgh, PA.

Abdel-Rahman, E. M., Nayfeh, A. H., Masoud, Z. N. (2003). "Dynamics and Control of Cranes: A Review". J. Vib. Control 9, 863-908.

Bartolini, G., Pisano, A., Usai, E. (2002). "Second-order sliding-mode control of container cranes". Automatica. 38, 1783-1790.

Cho, S. K., Lee, H. H. (2000). “An Anti-Swing Control of a 3-Dimensional Overhead Crane". in: Proceedings of the ACC, Chicago.

d'Andréa-Novel, B., Coron, J. M. (2000). "Exponential stabilization of an overhead crane with flexible cable via a back-stepping approach". Automatica. 36, 587-593.

Kang, S. C., Miranda, E. (2006). "Planning and Visualization for Automated Robotic Crane Erection Processes in Construction”. Autom. Constr. 15(4), 398-414.

Kang, S. C., Miranda, E. (2008). "Computational Methods for Coordinating Multiple Construction Cranes". J. Comput. Civil. Eng. 22(4), 252-263.

Kang, S. C., Chi, H. L., Miranda, E. (2009). "Three-Dimensional Simulation and Visualization of Crane Assisted Construction Erection Processes". J. Comput. Civil. Eng. 23(6), 363-371.

Lee, H. H. (2004). "A New Motion-Planning Scheme for Overhead Cranes With High-Speed Hoisting”. J. Dyn. Syst. Meas. Control. 126, 359-364.

Lee, H. H., Liang, Y., Segura, D. (2006). "A sliding-mode anti swing trajectory control for overhead cranes with high-speed load hoisting". J. Dyn. Syst. Meas. Control-Trans. ASME. $128,842-845$.

Sorensen, K. L., Singhose, W., Dickerson, S. (2007). "A controller enabling precise positioning and sway reduction in bridge and gantry cranes". Control. Eng. Pract. 15, 825-837. 